\title{
SÍNTESE E CARACTERIZAÇÃO DE FILMES DE NANOPARTÍCULAS DE PRATA DISPERSAS EM POLI(URETANO-UREIA) PARA PERMEAÇÃO DE GASES
}

\author{
A. C. C CAMPOS ${ }^{1}$, R. A. REIS ${ }^{1}$, C. VILANI ${ }^{2}$, D. R. G. LARRUDÉ ${ }^{2}$, M. D. DELPECH ${ }^{3}$ \\ ${ }^{1} \mathrm{UERJ} / \mathrm{IQ} / \mathrm{PPG}-\mathrm{EQ}$ \\ ${ }^{2} \mathrm{PUC}-\mathrm{RIO}$ \\ ${ }^{3} \mathrm{PPGQ} / \mathrm{IQ} / \mathrm{UERJ}$ \\ E-mail para contato: rdosreis@uerj.br
}

\begin{abstract}
RESUMO - Na permeação de gases, a modificação de polímeros pela adição de nanopartículas para formar membranas de transporte facilitado destaca-se dentre as opções promissoras. Os objetivos deste trabalho foram avaliar alterações químicas, estruturais e térmicas de um poli(uretano-ureia) (PUU) pela adição de nanopartículas de prata (AgNps) e obter dados de pemeabilidade de $\mathrm{CO}_{2}$. Alterações nos espectros na região do infravermelho nas bandas de estiramento das ligações $\mathrm{C}-\mathrm{O}-\mathrm{C}$ e $\mathrm{C}=\mathrm{O}$ e deslocamentos nos picos de difração obtidos por difração de raios $\mathrm{X}$ demonstram que houve interação entre as AgNps e o oxigênio éter do PUU. A interação polímero com AgNps diminuiu a estabilidade térmica dos domínios flexíveis, porém não alterou a decomposição inicial do polímero. As imagens de microscopia eletrônica de transmissão mostraram que houve baixa dispersão das AgNps. A presença das AgNps diminuiu a permeabilidade do $\mathrm{CO}_{2}$, mostrando a interferência dessas na sorção do gás. Foi proposto um mecanismo de interação entre as $\mathrm{AgNps}$ e o PUU e desse compósito com o $\mathrm{CO}_{2}$.
\end{abstract}

\section{INTRODUÇÃO}

O eteno é o principal insumo para a indústria petroquímica. De maneira geral, olefinas leves como eteno e propeno são obtidas através do craqueamento a vapor da nafta seguido por processos de destilação criogênica. Grande parte da energia utilizada nessa indústria é destinada ao processo de separação gasosa durante a destilação criogênica (Ren et al., 2008). Uma alternativa menos intensa em energia para promover tal separação está baseada nos processos de separação por membranas. Entretanto, para que esta tecnologia se torne viável, a membrana deve apresentar elevada seletividade para olefinas e deve se manter estável às condições de operação por um longo período de campanha (Bernado et al., 2009). Até o presente momento, as membranas desenvolvidas para a separação de olefinas e parafinas leves apresentaram baixa seletividade ou baixa estabilidade química, mantendo ainda desafiadora a busca por materiais mais promissores (Faiz e Li, 2012).

Membranas baseadas no mecanismo de sorção/difusão não são capazes promover a separação desejada entre olefinas e parafinas leves. Entretanto, a dispersão ao longo da matriz 


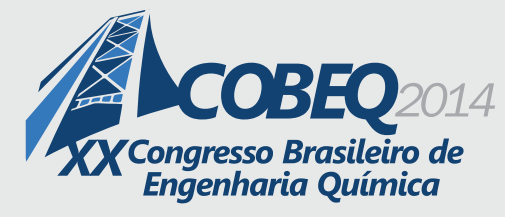

polimérica de carreadores que interajam preferencialmente com gases olefínicos, adiciona ao transporte um outro mecanismo denominado transporte facilitado, que aumenta consideravelmente a seletividade do processo de separação (Faiz e Li, 2012). Neste contexto, o uso de sais de prata como carreadores de olefinas tem produzido membranas poliméricas compósitas com seletividades promissoras. Entretanto a redução dos íons de prata $\left(\mathrm{Ag}^{+}\right)$ao longo da permeação leva a uma queda no desempenho dessas membranas, inviabilizando sua aplicação. Alternativamente, a utilização de nanopartículas de prata (AgNps) vem também sendo estudada para o transporte facilitado das olefinas, visando a síntese de membranas nanocompósitas mais estáveis. Como resultado, as propriedades de separação dos filmes poliméricos seriam mantidas em tempos maiores de operação (Kang et al., 2007). O transporte facilitado por AgNps está diretamente relacionado a sua dispersão na matriz polimérica e a ativação de sua superfície através de polarizadores adequados (Kang e Kang, 2011).

Os filmes de poli(uretano-ureia)s (PUUs) sintetizados a partir de dispersões aquosas possuem grupos com alta densidade eletrônica com potencial para não somente estabilizar a dispersão das AgNps em meio aquoso, como também ativá-las para o transporte facilitado de olefinas nos filmes formados a partir dessas dispersões. Os PUUs tem se destacado em estudos de permeação de gases (Barbosa et al., 2014). Neste contexto, o objetivo do presente trabalho é a síntese e caracterização de filmes compósitos de $\mathrm{AgNps}$ recobertas com polivinilpirrolidona (PVP) como agente estabilizante (4\% em massa) dispersos em matriz de PUU. Neste trabalho, pretende-se avaliar a distribuição das nanopartículas na matriz e sua influência nas características químicas e estruturais do filme. Visando entender as possíveis interações das AgNps com as cadeias de polímero, serão também obtidos dados de permeabilidade de $\mathrm{CO}_{2}$ e $\mathrm{N}_{2}$. O primeiro, por interagir com a cadeia do PUU, é mais sensível às alterações químicas do filme, enquanto o $\mathrm{N}_{2}$, por não apresentar tal interação, é mais sensível às alterações estruturais do caminho difusional (tamanho e distribuição de volume livre e mobilidade segmental).

\section{METODOLOGIA}

\subsection{Materiais}

Foram sintetizadas dispersões aquosas de poli(uretano-ureia)s à base de copolímero em bloco de poli(glicol etilênico) e poli(glicol propilênico) (EG-b-PG), com 7\% em massa de glicol etilênico, Dow Chemical Company, $\overline{\mathrm{M}} \mathrm{n}: 1850 \mathrm{~g} / \mathrm{mol}$; ácido dimetilolpropiônico (DMPA), Aldrich Chemical Company; etilenodiamina (EDA), 97\%, Acros Organics; diisocianato de isoforona (IPDI), doado pelo Centro Técnico Aeroespacial (CTA); poli(glicol propilênico) (PPG), Dow Chemical Company, $\overline{\mathrm{M}}$ : $1350 \mathrm{~g} / \mathrm{mol}$; trietilamina (TEA), Vetec Química Fina (Barbosa et al., 2014). Foram usados gases fornecidos pela Linde do Brasil: nitrogênio $\left(\mathrm{N}_{2}\right)$ com 99,9\% de pureza e gás carbônico $\left(\mathrm{CO}_{2}\right)$ com 99,9\% de pureza. As AgNps com 99,5\% de pureza em prata revestidas com PVP (4\% em massa) foram adquiridas da Sigma Aldrich.

\subsection{Síntese dos filmes}

O PUU foi sintetizado segunda a metodologia apresentada por Delpech e Miranda (2012). 


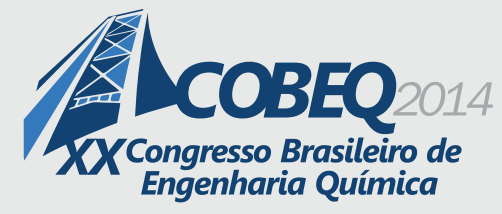

19 a 22 de outubro de 2014

Florianópolis/SC

Foi utilizada uma formulação de poliol com $25 \%$ de PPG e $75 \%$ de EG-b-PG e razão NCO/OH de 1,5 (Barbosa et al., 2014; Pereira, 2012). Uma dispersão aquosa de PUU com um teor de sólidos de $30 \%$ foi obtida, e em seguida diluída a um teor de sólidos de $6 \%$ para aumentar o tempo de estocagem. As AgNps em pó foram dispersas na dispersão de PUU por sonicação por 2 horas utilizando um misturador ultrassônico de alta frequência (modelo CPX 750 - Coler Parme). Quatro filmes de PUU/AgNps foram preparados evaporando-se a água da dispersão: PUU (branco), AgNps <0,2\%, AgNps 0,2\% e AgNps $1 \%(\mathrm{~m} / \mathrm{m})$. As porcentagem são relativas à quantidade de AgNps no filme compósito final de PUU/AgNps.

\subsection{Caracterização}

As analises na região do infravermelho foram realizadas num espectrômetro FT-IR da Perkin Elmer (Spectrum One), com acessório de refletância total atenuada e resolução de $4 \mathrm{~cm}^{-1}$ e 50 varreduras. Padrões de difração de raios $\mathrm{X}$ (DRX) com radiação de $\mathrm{K} \alpha$ do cobre foram obtidos a um passo de varredura de $0,05^{\circ}$ em difratômetro Rigaku Miniflex II. As micrografias de transmissão eletrônica foram obtidas utilizando um microscópio Jeol modelo JEM - 1210 operando a $80 \mathrm{kV}$. As análises de estabilidade térmica foram realizadas em um analisador termogravimétrico (Dp Union ${ }^{\circledR}$ Instrumentação analítica, modelo SDT Q 600). As amostras com massa entre 8 e $15 \mathrm{mg}$ foram colocadas em um cadinho de alumina sob atmosfera de $\mathrm{N}_{2}$ (fluxo de $20 \mathrm{~mL} / \mathrm{min}$ ). Uma taxa de aquecimento de $20^{\circ} \mathrm{C} / \mathrm{min}$ foi utilizada entre 25 e $600^{\circ} \mathrm{C}$. As temperaturas iniciais de degradação $\left(T_{\text {onset }}\right)$ dos filmes foram obtidas das curvas de termogravimetria derivativa (DTG). Uma tangente foi traçada nos pontos iniciais do primeiro pico das curvas. O valor no qual essa tangente cruza a abscissa é o valor de $T_{\text {onset. }}$.

\subsection{Testes de permeação}

Os dados de permeabilidade foram medidos através de um aparato de permeação por ganho de pressão (Romero et al., 2011) operado a 297,8 8 K e diferença de 3 bar através da membrana. Os dados de permeabilidade $(\boldsymbol{P})$ foram calculados pela a Equação 1, onde $d P_{p} / d t$ $\left(\mathrm{cmHg} . \mathrm{s}^{-1}\right)$ é a inclinação inicial da curva da pressão do permeado em função do tempo (em tempos iniciais de permeação), $V_{P}\left(\mathrm{~cm}^{3}\right)$ é o volume da câmara de permeado, $T(\mathrm{~K})$ é a temperatura do experimento, $\delta(\mathrm{cm})$ é a espessura do filme, $A_{m}\left(\mathrm{~cm}^{2}\right)$ é a área efetiva de membrana, $\Delta P(\mathrm{cmHg})$ é a diferença entre pressão na alimentação e a pressão no permeado no início do experimento. Foram feitos testes em duplicatas para cada filme.

$$
\boldsymbol{P}=\frac{273,15}{76} \frac{d P_{p}}{d t} \frac{V_{p} \delta}{T A_{m} \Delta P}
$$

\section{RESULTADOS E DISCUSSÕES}

As mudanças nas ligações de hidrogênio ocorridas no PUU pela adição de AgNps foram analisadas pela espectrometria FTIR. Observou-se um aumento na intensidade da banda stretching N-H (ligado) em $3332 \mathrm{~cm}^{-1}$ (Figura 1a), indicando um aumento do número de 


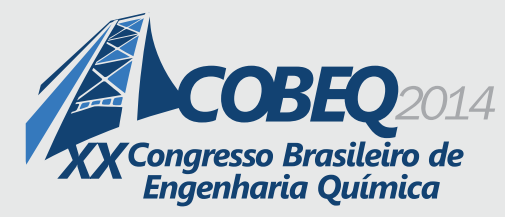

19 a 22 de outubro de 2014

Florianópolis/SC

doadores de prótons ( $\mathrm{N}-\mathrm{H}$ uretânico e ureico) comprometidos com ligações de hidrogênio. As bandas de carbonila uretânica ligada $(s t \mathrm{C}=\mathrm{O})$ e ureica ligada e ordenada $($ st $\mathrm{C}=\mathrm{O}$ ) tiveram suas frequências diminuídas (Figura 1b). Na região de carbonila, mudanças para frequências menores do espectro indicaram que ocorreu uma intensificação das ligações de hidrogênio realizadas por esse grupo. Pode-se dizer que essas carbonilas realizaram ligações de hidrogênio mais fortes, intensificando o ordenamento nos domínios rígidos do polímero. Logo, houve uma maior interação entre os grupos uretânicos e ureico, provocando assim, uma microseparação de fases entre os domínios rígidos e flexíveis. A mudança na região de amida II (Figura 1b) indicou uma alteração nas ligações de hidrogênio intermoleculares e na conformação da cadeia polimérica (Delpech, Miranda, 2012).
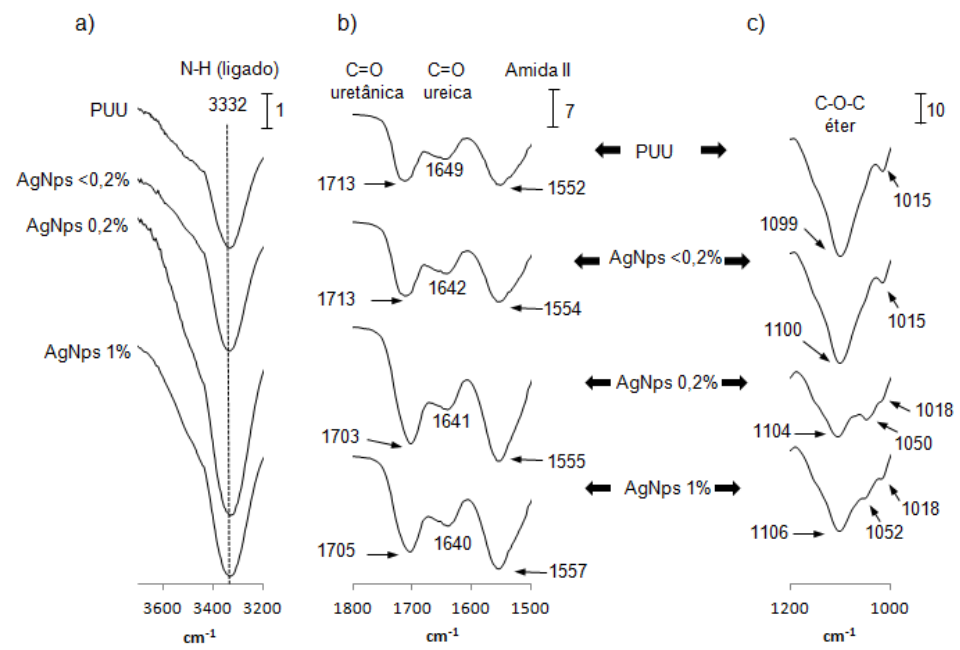

Figura 1 - Regiões do espectro de absorção IR onde ocorreram as principais mudanças com a adição das AgNps: (a) na região de N-H ligado; (b) na região de carbonila uretânica ligada, ureia ligada ordenada e de amida II; e (c) na região da banda de éter.

O surgimento de uma nova banda na região do grupo éter dos filmes PUU 0,2\% e 1\% sugere que os oxigênios éteres encontram-se em diferentes ambientes químicos (Figura 1c). A banda entre 1106-1104 $\mathrm{cm}^{-1}$ foi atribuída à interação mais fraca e a de menor número de onda $\left(1052-1050 \mathrm{~cm}^{-1}\right)$ a interação mais forte. Isso indicou um enfraquecimento das ligações de hidrogênio entre o oxigênio éter e a carbonila uretânica, justificando assim o deslocamento da banda já existente para maiores frequências. Esse enfraquecimento está sendo causado pela interação das $\mathrm{AgNps}$ com o oxigênio éter, que diminui a intensidade das interações desses grupos com as carbonilas (Feng et al., 2013). A banda em 1052-1050 $\mathrm{cm}^{-1}$ aparenta surgir por causa da interação direta entre o oxigênio éter e as AgNps (Kang et al., 2008).

As AgNps apresentaram o mesmo perfil de DRX observado no trabalho de Lanje et al. (2010). Foi possível identificar os picos de difração em valores $2 \theta$ de $38,05^{\circ}$ e $44,02^{\circ}$ correspondentes aos planos $\left(\begin{array}{lll}1 & 1 & 1\end{array}\right)$ e $\left(\begin{array}{lll}2 & 2 & 0\end{array}\right)$ de cristais de prata (Figura 2). No filme AgNps 1\%, os picos foram deslocados $0,50^{\circ}$, enquanto no filme $\operatorname{AgNps} 0,2 \%$ o pico $\left(\begin{array}{lll}1 & 1 & 1\end{array}\right)$ foi deslocado 


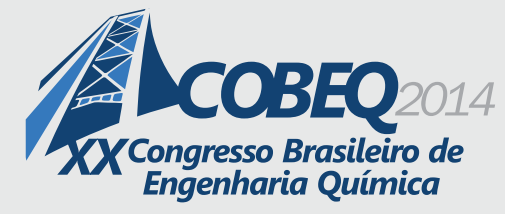

19 a 22 de outubro de 2014

Florianópolis/SC

$0,60^{\circ}$. Nesses filmes a interação entre o polímero e as AgNps conseguiu modificar a estrutura cristalina das AgNps (Bellido, Assaf, 2009). Considerando as análises de FTIR, pode-se dizer que essa modificação estrutural foi provocada pela interação entre as AgNps e o oxigênio éter. Não foi possível encontrar picos de prata no filme $\mathrm{AgNps}<0,2 \%$, pois a quantidade de AgNps foi pequena para detecção através dessa técnica.

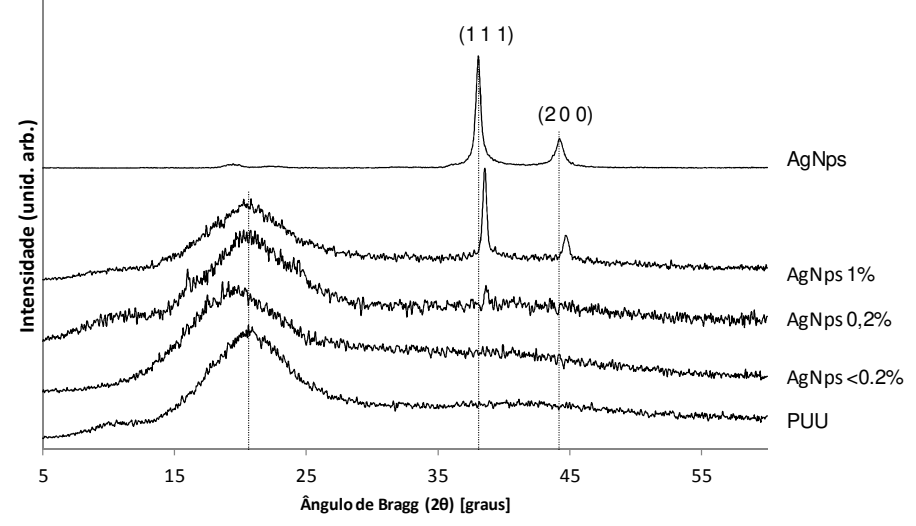

Figura 2 - Difratogramas dos filmes preparados e das AgNps

Analisando as micrografias de TEM (Figura 3), fica claro que as AgNps tiveram uma baixa dispersão na matriz polimérica. Os filmes não se mostraram homogêneos, com AgNps concentradas em poucas regiões do material. No filme AgNps <0,2\% (Figura 3a), as AgNps estavam dispostas em pequenas regiões com tamanho de cerca de $190 \mathrm{~nm}$. Elas não se aglomeraram, permanecendo com tamanho inferior a $20 \mathrm{~nm}$. Esse filme foi o único que apresentou coloração amarelada, os demais filmes com AgNps apresentaram uma aspecto escuro. A coloração amarela é característica de AgNps encontradas com tamanho inferior a 100 nm e não aglomeradas. Essa coloração é estendida a filmes de PU que contém AgNps nessa dimensão (Wu et al., 2009). Embora as AgNps não estivessem bem distribuídas, as poucas regiões contendo prata foram suficiente para fornecer essa coloração amarela ao filme. No filme AgNps 0,2\% (Figura 3b) e AgNps 1\% (Figura 3c), as AgNps encontradas estavam dispostas em regiões bem maiores. Diferente do filme $\mathrm{AgNps}<0,2 \%$, nessas regiões foram formados grandes aglomerados contendo AgNps com tamanho superior a $100 \mathrm{~nm}$.

Quanto ao perfil de degradação dos filmes, não ocorreu nenhuma mudança significativa na $T_{\text {onset }}\left(205^{\circ} \mathrm{C}\right)$ em relação ao filme de PUU. A $T_{\text {onset }}$ está relacionada à degradação dos domínios rígidos do polímero (Coutinho et al., 2003). Isso evidenciou que as mudanças provocadas no PUU com a adição de AgNps não influenciaram a degradação dos grupamentos ureicos e uretânicos presentes nos domínios rígidos. Com isso, a resistência térmica do polímero foi mantida. Por outro lado, houve mudança na temperatura de máxima velocidade de perda de massa $\left(T_{\text {vmáx }}\right)$ em comparação ao filme de PUU. A $T_{\text {vmáx }}$ está relacionada à degradação dos domínios flexíveis do polímero (Coutinho et al., 2003). Verificou-se uma queda na $T_{\text {vmáx }}$, reforçando a hipótese sugerida pelas outras análises que os domínios flexíveis dos polímeros estão sofrendo alteração com a presença das $\mathrm{AgNps}$ devido as novas interações formadas. A 


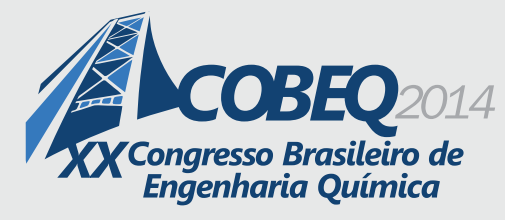

19 a 22 de outubro de 2014

Florianópolis/SC

interação das AgNps com o oxigênio éter enfraqueceu as ligações de hidrogênio entre grupo éter e N-H uretânico, a medida que elas enfraqueceram, a temperatura de $T_{\text {vmáx }}$ também diminuiu (Cakic et al., 2009; Coutinho et al., 2003).

a)

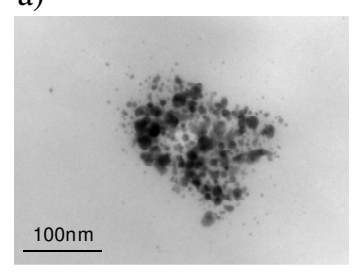

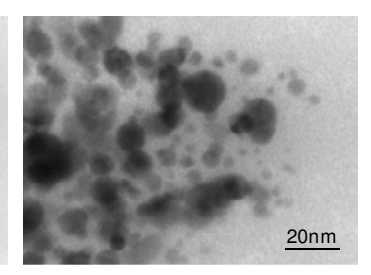

c) b)
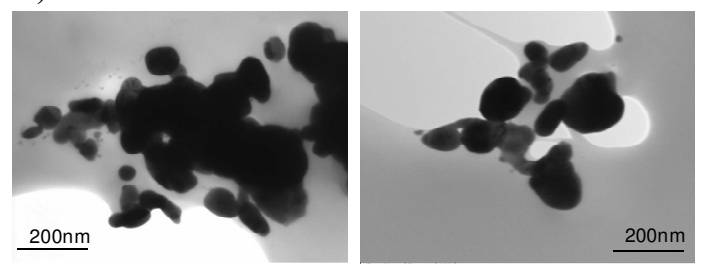

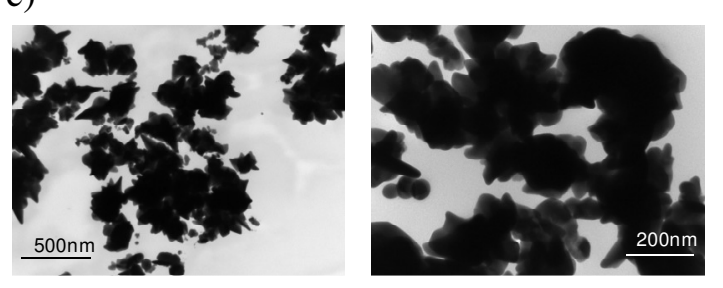

Figura 3 - Imagens de TEM dos filmes. Teores de AgNps dos filmes: a) <0,2\%; b) 0,2\% e C) $1 \%$.

Os valores de permeabilidade de $\mathrm{CO}_{2}$ e $\mathrm{N}_{2}$ em função do teor de $\mathrm{AgNps}$ estão contidos na Tabela 1. $\mathrm{O} \mathrm{CO}_{2}$ possui afinidade pelo PUU devido à interação com os grupos éteres presentes na estrutura (Lin e Freeman, 2004). O transporte das moléculas acontece através dos domínios flexíveis do PUU, onde estão localizados os grupos éteres (Lau et al., 2013). Os filmes poliméricos preparados com a adição de AgNps tiveram em geral uma queda acentuada de permeabilidade do $\mathrm{CO}_{2}$ seguida por uma diminuição um pouco mais branda na permeabilidade do $\mathrm{N}_{2}$. A queda na permeabilidade do $\mathrm{CO}_{2}$ deve-se principalmente a interação entre as AgNps adicionadas e o oxigênio do grupo éter. Essa interação com as AgNps diminui a disponibilidade desses grupos para interagir com o $\mathrm{CO}_{2}$, diminuindo assim sua solubilidade na matriz polimérica. A queda na permeabilidade do $\mathrm{N}_{2}$ está possivelmente ligada a dois efeitos: (i) diminuição da mobilidade segmental dos segmentos flexíveis do polímero provocada pela interação com a AgNps que restringem o movimento das cadeias poliméricas; e (ii) a formação de agregados de Nps, mostrados nas imagens de TEM, que aumentariam a tortuosidade do caminho difusional na matriz. Esses dois motivos também interferiram na permeabilidade $\mathrm{CO}_{2}$, diminuindo o coeficiente de difusão de ambos, porém o efeito na sorção é mais pronunciado neste sistema.

Assim como o $\mathrm{CO}_{2}$, olefinas leves também apresentam interações específicas com os grupos polares da estrutura de PUU (Lin e Freeman, 2004). Considerando apenas o mecanismo de sorção/difusão, não seria surpresa que a adição de AgNps comprometesse a permeabilidade das olefinas de forma mais pronunciada que a das parafinas. Por outro lado, as AgNps podem agir como carreador para o transporte facilitado compensando assim a perda da interação entre o oxigênio éter do PUU e as olefinas. A interação entre as AgNps e o oxigênio por sua vez, pode 
polarizar a superfície das Nps ativando-as para o transporte facilitado de olefinas. Logo os filmes de PUU/ AgNps possuem potencial para atuar como material para o preparo de membranas para a separação olefinas/parafinas leves.

Tabela 1 - Permeabilidade de $\mathrm{CO}_{2}$ e $\mathrm{N}_{2}$ nos filmes de PUU/AgNps em função do teor de AgNps a $297 \pm 2 \mathrm{~K}$.

\begin{tabular}{|c|c|c|}
\hline \multirow[t]{2}{*}{ Filme } & \multicolumn{2}{|c|}{ Permeabilidade (barrer ${ }^{a}$ ) } \\
\hline & $\mathrm{CO}_{2}$ & $\mathrm{~N}_{2}$ \\
\hline PUU & 180 & 3,80 \\
\hline AgNps $<0,2 \%$ & 134 & 3,56 \\
\hline AgNps 0,2\% & 83,8 & 1,70 \\
\hline AgNps $1 \%$ & 72,5 & 1,59 \\
\hline
\end{tabular}

\section{CONCLUSÕES}

Nos filmes de PUU/AgNps sintetizados nesse trabalho, as nanocargas interagiram preferencialmente com os oxigênios éteres presentes na estrutura polimérica, o que compromete a interação favorável PUU e o $\mathrm{CO}_{2}$. A resistência térmica do PUU foi mantida com a adição das nanopartículas. A interação entre o oxigênio éter e as AgNps poderá ser explorada na sequência do trabalho. Outras estratégias para melhorar a dispersão das AgNps na matriz polimérica poderão ser abordadas, tornando assim os filmes de PUU/AgNps promissores para o transporte facilitado de olefinas.

\section{REFERÊNCIAS}

BARBOZA, E.M.; DELPECH, M. C.; GARCIA, M. E. F.; PIMENTA, F. D. Avaliação das Propriedades de Barreira de Membranas Obtidas a partir de Dispersões Aquosas à Base de Poliuretanos e Argila. Polímeros (São Carlos. Online), v. 24, p. 94-100, 2014.

BELLIDO, J. D. A.; ASSAF, E. M. Reforma seca de metano sobre catalisadores de níquel suportados em $\mathrm{CeO}_{2}$ modificado com $\mathrm{MgO}$. Anais do $5^{\circ}$ Congresso brasileiro de pesquisa e desenvolvimento em petróleo e gás, Fortaleza, Brasil, 2009.

BERNARDO, P.; DRIOLI, E; GOLEMME, G. Membrane Gas Separation: A Review/State of the Art. Ind. Eng. Chem. Res., v. 48, p. 4638-4663, 2009.

CAKIC, M. S.; STAMENKOVIC, J. V.; DJORDJEVIC, D. M.; RISTIC, I. S. Synthesis and degradation profile of cast films of PPG-DMPA-IPDI aqueous polyurethane dispersions based on selective catalysts. Polym. Degrad. Stab., v. 94, p. 2015-2022, 2009.

COUTINHO, F. M. B.; DELPECH, M. C.; ALVES, T. L., FERREIRA, A. A. Degradation profile of cast films of polyurethane and poly(urethane-urea) aqueous dispersions based on hydroxyl-terminated polybutadiene and different diidocyanates. Polym. Degrad. Stab., v. 81, p. 19-27, 2003. 


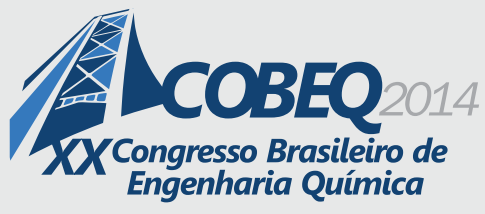

DELPECH, C. M.; MIRANDA, G. S. Waterborne polyurethanes: influence of chain extender in ftir spectra profiles. Cent. Eur. J. Eng., v. 2, p. 231-238, 2012.

FAIZ, R; LI, K. Olefin/paraffin separation using membrane based facilitated transport/chemical absorption techniques. Chem. Eng. Sci., v. 73, p. 261-284, 2012.

FENG, S.; REN, J.; HUA, K.; LI, H.; REN, X.; DENG, M. Poly(amide-12-b-ethylene oxide)/polyethylene glycol blend membranes for carbon dioxide separation. Sep. Purif. Technol., v. 116, p. 25-34, 2013.

KANG, S. W; HONGA, J.; PARK, J. H.; MUNC, S. H.; KIM, J. H.;CHOE, J.; CHARA, K.; KANG, Y. S. Nanocomposite membranes containing positively polarized gold nanoparticles for facilitated olefin transport. J. Membr. Sci., v. 321, p.90-93, 2008.

KANG, S. W.; KANG, Y. S. Silver nanoparticles stabilized by crosslinked poly(vinyl pyrrolidone) and its application for facilitated olefin transport. J. Membr. Sci., v. 353, p. 83-86, 2011.

KANG, Y. S.; KANG, S. W.; KIM, H.; KIM, J. H.; WON, J.; KIM, C. K.; CHAR, K. Interaction with olefins of the partially polarized surface of silver nanoparticles activated by $\mathrm{p}$ benzoquinone and its implications for facilitated olefin transport. Adv. Mater., v. 19, p. 475-479, 2007.

LANJE, A. S.; SHARMA, S. J.; PODE, R. B. Synthesis of silver nanoparticles: a safer alternative to conventional antimicrobial and antibacterial agents. J. Chem. Pharm., v. 2, p. 478-483, 2010.

LAU, C. H.; LI, P.; LI, F.; CHUNG, T. S.; PAUL, D. R. Reverse-selective polymeric membranes for gas separations. Prog. Polym. Sci., v. 38, p. 740-766, 2013.

LIN, H.; FREEMAN, B. D. Gas solubility, diffusivity and permeability in poly(ethylene oxide). J. Membr. Sci., v. 239, p 105-117, 2004.

PEREIRA, J. H. C. Caracterização de filmes formados por dispersões aquosas de poli(uretanoureia)s para aplicação em membranas para permeação de gases. 2012. 88f. Dissertação (Mestrado em Engenharia Química) - Instituto de Química, Universidade Estadual do Rio de Janeiro, Rio de Janeiro, 2012.

REN, T; PATEL, M. K.; BLOK, K. Steam cracking and methane to olefins: Energy use, $\mathrm{CO}_{2}$ emissions and production costs. Energy, v. 33, p. 817-833, 2008.

ROMERO, A. I.; PARENTIS, M. L.; HABERT, C. H.; GONZO, E. E. Synthesis of polyetherimide/silica hybrid membranes by the sol-gel process: influence of the reaction conditions on the membrane properties. J. Mat. Sci., v. 46, p. 4701-4709, 2011.

WU, C. I.; HUANG, J. W.; WEN, Y. L.;WEN, S. B.; SHEN, Y. W.; MOU-YUNG YEH, M. Y. Preparation of antibacterial waterborne polyurethane/silver nanocomposite. J. Chin. Chem. Soc., v. 56, p. 1231-1235, 2009. 